\title{
Production et valeur nutritive de fourrages de pois Mascate (Mucuna pruriens (L.) DC. var. utilis (Wall. ex Wight) Baker ex Burck) cv. Ghana cultivé dans le Centre et Nord bassin arachidier sénégalais
}

\author{
Ambroise DIATTA ${ }^{1 *}$, Abdoulaye DIENG ${ }^{2}$, Mamadou Tandiang DIAW ${ }^{2}$, \\ Thierry Daniel Tamsir NESSEIM ${ }^{2}$ et Saliou NDIAYE ${ }^{3}$ \\ ${ }^{1}$ Institut Sénégalais de Recherches Agricoles - Centre de Recherches Zootechniques (ISRA-CRZ), BP 53 \\ Kolda, Sénégal. \\ ${ }^{2}$ Département de Productions Animales, Université de Thiès - Ecole Nationale Supérieure d'Agriculture (UT- \\ ENSA), Route de Khombole, BP A296 Thiès, Sénégal. \\ ${ }^{3}$ Département de Productions Végétales, Université de Thiès - Ecole Nationale Supérieure d'Agriculture (UT- \\ ENSA), Route de Khombole, BP A296 Thiès, Sénégal. \\ *Auteur correspondant ; E-mail : amboudiatta58@yahoo.fr, Tél : 00221775017684.
}

\section{REMERCIEMENTS}

Les auteurs adressent leurs sincères remerciements à la Banque Mondiale (BM) pour avoir financé cette étude à travers le West African Agricultural Productivity Program/Programme de Productivité Agricole en Afrique de l'Ouest (WAAPP/ PPAAO).

\section{RESUME}

L'insuffisance des ressources fourragères naturelles constitue une contrainte majeure au développement de l'élevage des ruminants dans le Centre et Nord bassin arachidier sénégalais. Pour y faire face, il faut recourir à des techniques telles que la culture fourragère. L'objectif est donc d'étudier la production de fourrage de Mucuna pruriens dans la zone et de déterminer sa valeur nutritive. Du fourrage frais de 79-84 jours et des foins de 60, 75 et 90 jours ont été produits. Les rendements ont été mesurés, les fourrages analysés, les compostions chimiques et les valeurs nutritives déterminées. Les rendements sont globalement faibles, 1,16 à 1,50 t MS/ ha, mais comparables à certains rapportés dans la littérature. Le fourrage frais est plus riche en protéines brutes que les foins, soit 19,56 contre 14,16 à 18,65\% de la MS, moins fibreux, soit 27,26 contre 33,82 à 35,27\% de la MS et plus nutritif, soit 150,65 contre 96,60 à $141,48 \mathrm{~g} / \mathrm{kg}$ MS de protéine brute digestible et 0,70 contre 0,67 à 0,69 $\mathrm{UF} / \mathrm{kg}$ MS. Parmi les foins, celui de 75 jours offre un léger avantage. En définitive, cette culture reste possible dans cette zone et peut y être préconisée en vue d'une atténuation du déficit fourrager.

(C) 2020 International Formulae Group. All rights reserved.

Mots clés : Rendement, composition chimique, fourrage frais, foins, valeur azotée, valeur énergétique.

\section{Production and nutritional value of Muscat pea fodder (Mucuna pruriens (L.) DC. var. utilis (Wall. ex Wight) Baker ex Burck) cv. Ghana cultivated in the Center and North senegalese groundnut basin}

\begin{abstract}
The insufficiency of natural fodder resources constitutes a major constraint to the development of ruminant farming in the Central and Northern senegalese groundnut basin. To deal with this, techniques such as forage cultivation must be used. The objective is therefore to study the forage production of Mucuna pruriens in
\end{abstract}


the area and determine its nutritional value. Fresh forage of 79-84 days and hay of 60, 75 and 90 days were produced. Yields were measured, forages analyzed, chemical compositions and nutrient values determined. Yields are generally low, 1.16 to $1.50 \mathrm{t} \mathrm{DM} /$ ha, but comparable to some reported in the literature. Fresh forage is richer in crude protein than hay, 19.56 against 14.16 to $18.65 \%$ DM, less fibrous, 27.26 against 33.82 to $35.27 \%$ DM and more nutritious, 150.65 against 96.60 to $141.48 \mathrm{~g} / \mathrm{kg} \mathrm{DM}$ of digestible crude protein and 0.70 against 0.67 to $0.69 \mathrm{UF} / \mathrm{kg}$ DM. Among the hays, that of 75 days offers a slight advantage. Ultimately, this crop remains possible in this area and can be recommended there with a view to reducing the fodder deficit.

(C) 2020 International Formulae Group. All rights reserved.

Keywords: Yield, chemical composition, fresh fodder, hay, nitrogen value, energy value.

\section{INTRODUCTION}

Le pois Mascate (Mucuna pruriens (L.) DC. var. utilis (Wall. ex Wight) Baker ex Burck) est une légumineuse herbacée de la famille des Fabaceae et du genre Mucuna. Ce genre compte une centaine d'espèces dont Mucuna pruriens. Cette espèce comprend deux (2) variétés, Mucuna pruriens var. pruriens, la variété sauvage et Mucuna pruriens var. utilis, la variété cultivée. Actuellement, on considère que toutes les formes cultivées ou cultivars, dont cv. Ghana, proviennent de la variété utilis (St-Laurent et al., 2002; Padmesh et al., 2006; Dahouda, 2009).

Mucuna pruriens serait originaire de l'Asie du Sud et de la Malaisie et est actuellement très répandu sous les tropiques. Il a besoin d'un climat chaud et humide, d'une pluviométrie annuelle comprise entre 650 et $2500 \mathrm{~mm}$, pour bien se développer. Il peut pousser du niveau de la mer jusqu'à une altitude de $2100 \mathrm{~m}$. Il s'accommode de sols très divers, sableux à argileux et supporte une acidité assez élevée. M. pruriens var. utilis est surtout cultivé à travers le monde comme engrais vert, plante de couverture, améliorante des jachères, antiérosive, ou pour lutter contre les adventices telles que Imperata cylindrica (L.) P. Beauv, Striga hermonthica (Del.) Benth. Il est aussi cultivé pour son fourrage en pur ou en association avec des céréales comme le maïs et le sorgho. Les graines et les gousses vertes sont consommées par certaines populations ou utilisées comme concentré en alimentation animale. Sur le plan médicinal, Mucuna est utilisé dans le traitement de la maladie de Parkinson (Dahouda, 2009; Coulibaly et al., 2012, 2018; Heuzé et al., 2015; ECHO, 2020).
Au Sénégal, les recherches fourragères sur le Mucuna ont démarré en 1962 à la station de Séfa en Moyenne Casamance (région de Sédhiou) au Sud du pays. Elles ont permis, entre autres, d'identifier une variété appelée Mucuna «Noir de Séfa ». Les recherches se sont ensuite poursuivies et étendues de manière discontinue dans le Sud du pays. Dans les années 2000, sept (7) variétés ou cultivars dont le cultivar Ghana, en provenance de l'Educational Concerns for Hunger Organization (ECHO), de l'International Livestock Research Institute (ILRI), de l'International Institute for Tropical Agriculture (IITA) et du Centre d'Information et d'Echange sur les Plantes de Couverture en Afrique (CIEPCA), ont été introduits au Sénégal en tant que plantes de couverture. Suite à cette introduction, les recherches fourragères sur le Mucuna ont repris dans le sud du pays, élargissant ainsi la liste des légumineuses fourragères annuelles en étude au Sénégal. En effet, avec la réduction de plus en plus importante des aires de parcours et subséquemment des ressources fourragères naturelles pour tout le pays en général et le Centre et Nord bassin arachidier sénégalais (CNBAS) en particulier, il faut recourir à des techniques plus productrices de fourrage et pouvant s'insérer harmonieusement dans les systèmes de cultures pratiqués dans les différentes zones agricoles du pays. La présente étude s'inscrit dans ce cadre. Elle a ainsi pour objectif d'étudier la production fourragère $M$. pruriens cv. Ghana dans le CNBAS et de déterminer la valeur nutritive du fourrage en vue de contribuer à la sécurité alimentaire des ruminants domestiques par une disponibilité accrue du fourrage de cette légumineuse. 


\section{MARTERIEL ET METHODES Zone d'étude}

La zone d'étude (CNBAS) correspond à la région de Thiès et fait partie d'une zone largement plus vaste, le bassin arachidier sénégalais (BAS). Cette région de Thiès se situe entre $14^{\circ} 46^{\prime} 58$ " de latitude Nord et 16 - 54 ' 06 " de longitude Ouest et s'étend sur $6601 \mathrm{~km}^{2}$, soit 3,4\% du territoire national. Sur le plan climatique, la région est sous influence des alizés maritimes et de l'harmattan et présente un climat de type soudano sahélien. Selon l'Agence Nationale de l'Aviation Civile et de la Météorologie (ANACIM) de Thiès, la région a enregistré $503 \mathrm{~mm}$ en 2018 contre des moyennes annuelles (1998-2007) qui ont varié entre 400 et $600 \mathrm{~mm}$ pour l'ensemble du BAS (Dione et al., 2008). Les sols sont de plusieurs types, avec cependant une nette prédominance des sols ferrugineux tropicaux. De manière plus précise, la production de fourrage a été réalisée dans les parcelles d'expérimentations agronomiques du Centre d'Application des Techniques Agricoles (CATA) de l'Ecole Nationale Supérieure d'Agriculture $\left(14^{\circ} 16^{\prime}\right.$ $\left.\mathrm{N} \& 16^{\circ} 57^{\prime} \mathrm{W}\right)$ de l'Université de Thiès (ENSA/UT)

\section{Matériel végétal}

Le matériel végétal était constitué de semences de $M$. pruriens $\mathrm{cv}$. Ghana produites durant la campagne 2017-2018 à la station de recherches du Centre de Recherches Zootechniques de Kolda (CRZK) de l'Institut Sénégalais de Recherches Agricoles (ISRA).

\section{Conduite des cultures}

Deux (2) parcelles de $300 \mathrm{~m} \times 40 \mathrm{~m}$ chacune délimitées par trois (3) brise-vents ont été choisies pour abriter les cultures. Dans l'une, une sous-parcelle de $2775 \mathrm{~m}^{2}(75 \mathrm{~m} \mathrm{x}$ $37 \mathrm{~m}$ ) a été délimitée et a servi à la production de fourrage frais. Elle a été ceinturée par une allée de $3 \mathrm{~m}$ de large. Dans l'autre parcelle, trois (3) sous-parcelles de $2176 \mathrm{~m}^{2}$ (68 m x 32 $\mathrm{m})$ chacune ont été délimitées et ont servi à la production des foins âgés respectivement de 60,75 et 90 jours. Elles ont été ceinturées et séparées entre elles par des allées également larges de $3 \mathrm{~m}$. Les sous-parcelles ont été défrichées et labourées à sec à l'aide d'un tracteur équipé d'un pulvériseur à disques. Après le travail du sol, le semis a été effectué à $\mathrm{sec}$, en lignes espacées de $100 \mathrm{~cm}$ et sur les lignes, en poquets distants également de 100 $\mathrm{cm}$. Trois (3) graines ont été semées par poquet. Les semences ont été préalablement traitées avec du granoforce rouge [Perméthrine $(25 \mathrm{~g} / \mathrm{kg})+$ Thiram $(250 \mathrm{~g} / \mathrm{kg})]$ à la dose de 10 $\mathrm{g} / 8 \mathrm{~kg}$ de semences. Après les semis, un traitement du sol a été effectué avec du carbofuran (furadan $5 \% \mathrm{G}$ ) à la dose de 3 $\mathrm{kg} /$ ha et de l'engrais NPK 6-20-10 a été aussi épandu à la volée à la dose de $150 \mathrm{~kg} / \mathrm{ha}$. Des travaux d'entretien ont été réalisés, notamment avec un sarclo-binage, du $18^{\mathrm{eme}}$ au $27^{\mathrm{eme}}$ jour et un démariage à deux (2) plants par poquet à partir du $33^{\text {eme }}$ jour après semis en vue de respecter la densité de peuplement de 20000 plants/ ha. Après le semis à sec, la $1^{\text {ère }}$ pluie utile (pluie ayant permis la levée) a été enregistrée le 23 août 2018. Cette date a ainsi été considérée comme date de semis.

\section{Evaluation des rendements en fourrage}

Les rendements en fourrage ont été évalués selon la méthode des carrés de rendements. Dix (10) placeaux, de $4 \mathrm{~m}^{2}(2 \mathrm{~m} \mathrm{x}$ $2 \mathrm{~m})$ chacun, ont été choisis de manière aléatoire dans chacune des sous-parcelles de production par l'utilisation de la table des nombres aléatoires (Dagnelie, 1998). Le fourrage de chaque placeau a été fauché à la faucille et pesé avec une balance électronique de $10 \mathrm{~kg}$ de portée. Après la pesée, un échantillon de $500 \mathrm{~g}$ de fourrage a été constitué par placeau et séché à l'étuve à $105^{\circ} \mathrm{C}$ jusqu'à poids constant durant 48 heures. A la sortie de l'étuve, l'échantillon a été de nouveau pesé et le taux de matière sèche $(\% \mathrm{MS})$ et le rendement (t MS/ ha) ont été ensuite déterminés par calcul suivant les formules ciaprès :

$\mathrm{MS}(\% \mathrm{MS})=(\mathrm{PF}-\mathrm{PS}) / \mathrm{PF} \times 100$;

Rendement (t MS/ha) $=(\mathrm{P} \times \mathrm{MS}) / 10 \times \mathrm{S}$; avec $\mathrm{PF}=$ poids frais de l'échantillon (kg); PS $=$ poids sec de l'échantillon au sortir de l'étuve $(\mathrm{kg}) ; \mathrm{P}=$ poids de fourrage récolté dans un placeau $(\mathrm{kg}) ; \mathrm{S}=$ surface du placeau $\left(\mathrm{m}^{2}\right)$.

\section{Fourrages produits}

Quatre (4) types de fourrages ont été produits et ont servi aux études sur la valeur 
alimentaire : il s'agit du fourrage frais âgé de 79 à 84 jours (fauché quotidiennement le matin durant 6 jours) et des fourrages secs (conservés en foins) récoltés respectivement à 60,75 et 90 jours. Les stades de développement étaient floraison-début fructification pour le foin de 60 jours et fructification pour les foins de 75 et 90 jours et le fourrage frais de 79 à 84 jours. Les foins ont été obtenus à la suite de fauches manuelles à l'aide de faucilles et de séchage naturel au champ sans retournement. Après séchage, les foins ont été transportés successivement au moyen d'une charrette à l'âne. Ils ont été stockés en vrac et séparément dans un magasin de fourrage du Centre d'Application des Techniques d'Elevage (CATE) de l'ENSA.

\section{Constitution des échantillons de fourrage}

Les études sur la valeur alimentaire de chaque fourrage (ingestion, digestibilité) ont comporté trois (3) phases successives d'adaptation (7 jours), de pré-expérimentation (7 jours) et d'expérimentation (6 jours). Pour chaque fourrage, un (1) échantillon a été constitué quotidiennement durant la phase d'expérimentation, soit un total de six (6) échantillons. Vingt quatre (24) échantillons ont ainsi été constitués pour l'ensemble des quatre (4) fourrages. Les échantillons de fourrage frais ont été séchés à l'air libre et à l'ombre alors que ceux des foins ont été constitués à partir des foins séchés naturellement au champ et stockés en vrac dans le magasin de fourrage du CATE.

\section{Constitution des échantillons pour les analyses}

Après le broyage des vingt quatre (24) échantillons, deux (2) groupes d'échantillons par type de fourrage ont été constitués, le Groupe 1 (G1) comprenant les trois (3) échantillons des jours 1, 2 et 3 (J1, J2 et J3) et le Groupe 2 (G2) ceux des jours 4, 5 et 6 (J4, J5 et J6). Pour chaque groupe, un échantillon de $100 \mathrm{~g}$ a été constitué à partir des poids relatifs des trois (3) échantillons constitutifs. De l'échantillon de chaque groupe, trois (3) échantillons finaux ont été constitués et utilisés pour les différentes analyses, soit un total de vingt quatre (24) échantillons finaux pour l'ensemble des quatre (4) types de fourrages.

\section{Analyse des échantillons}

Les analyses chimiques ont été effectuées au laboratoire de bromatologie de l'ENSA de Thiès suivant la méthode AOAC (1995). La matière sèche (MS) a été déterminée par la méthode 934.01, la matière organique (MO) et les cendres totales (CT) par la méthode 942.05 et la cellulose brute (CB) par la méthode 978.10. L'azote $(\mathrm{N})$ a été dosé selon la méthode Kjeldahl (AOAC, 1995). Les fibres, neutral detergent fiber (NDF) et acid detergent fiber (ADF), ont été dosées suivant la méthode Van Soest (1965) alors que la matière grasse (MG) ou extrait éthéré (EE) a été dosée sur le Rafatec. La protéine brute (PB) ou matière azotée totale (MAT) et l'extractif non azoté (ENA) ont été déterminés à l'aide des formules ci-après :

$\mathrm{PB}(\% \mathrm{MS})=\mathrm{N} \times 6,25$;

$\mathrm{ENA}(\% \mathrm{MS})=\mathrm{MO}-(\mathrm{PB}+\mathrm{CB}+\mathrm{EE})$;

avec $\mathrm{MO}, \mathrm{PB}, \mathrm{CB}$ et $\mathrm{EE}$ en pour-cent de la $\mathrm{MS}$ (\% MS).

\section{Détermination de la valeur nutritive des fourrages}

La valeur azotée, exprimée en protéine brute digestible (PBD) et la valeur énergétique, exprimée en unités fourragères (UF) ont été déterminées respectivement par les formules préconisées par Rivière (1978) pour les fourrages de graminées et de légumineuses et Dijskstra (1957) pour les légumineuses :

PBD $(\%$ MS $)=$ PB $-4,50$;

$\mathrm{UF}(/ \mathrm{kg}$ MS $)=(0,7882 \times(100-\mathrm{CT})-(0,1044$ $\mathrm{x} \mathrm{CB})-\left(2,551 \times \mathrm{CB}^{2}\right) /(100-\mathrm{CT}) \times 0,0143+$ $0,0375) / 100$;

avec $\mathrm{PB}, \mathrm{CT}$ et $\mathrm{CB}$ en pour-cent de la MS (\% MS).

\section{Analyse statistique des données}

Les données ont été saisies avec le logiciel Microsoft Excel 2007. Les analyses statistiques (statistique descriptive, ANOVA et méthode de Tukey au seuil de 5\%) ont été réalisées avec le logiciel Statistix 8.1. Ces analyses ont porté sur les rendements en fourrage et en valeurs azotée (PBD) et énergétique (UF), les teneurs en nutriments (MS, CT, MO, PB, CB, NDF, ADF, EE et ENA) et les valeurs nutritives (PBD et UF). 


\section{RESULTATS \\ Rendements en fourrage}

Les rendements moyens de fourrage frais (79-84 jours) et de fourrages secs (ou foins) âgés de 60, 75 et 90 jours sont consignés dans le Tableau 1. Les rendements ne montrent aucune différence significative $(\mathrm{p}=0,45)$ malgré des temps de croissance (semis-fauche) différents. Entre le foin de 60 jours et les foins de 75 et 90 jours, les rendements n'ont augmenté respectivement que de 28,45 et $29,31 \%$. Entre les foins de 75 et de 90 jours, l'augmentation n'a été que de $7 \%$.

\section{Compositions chimiques des fourrages}

Les teneurs moyennes en nutriments des fourrages sont consignées dans le Tableau 2. D'une manière générale, les teneurs en nutriments des quatre (4) types de fourrage ont varié significativement ( $\mathrm{p}<0,05)$. Les teneurs en PB sont toutes différentes significativement avec des teneurs plus élevées et plus basses respectivement pour le fourrage frais et le foin de 90 jours. Pour la CB, NDF et ADF, des différences significatives sont notées entre le fourrage frais et les foins. Entre les foins, aucune différence significative n'est observée. Les teneurs en $\mathrm{CB}$ et en $\mathrm{ADF}$ du fourrage frais sont moins élevées que celles des foins. Et c'est l'inverse pour les teneurs en NDF. Les temps de croissance n'ont pas entraîné des hausses significatives des teneurs en $\mathrm{CB}$ et $\mathrm{ADF}$ ni des baisses significatives en NDF des foins.

\section{Valeur nutritive des fourrages}

Les valeurs azotée et énergétique moyennes des fourrages, exprimées respectivement en protéines brutes digestibles (PBD) et unités fourragères (UF) sont consignées dans le Tableau 3. Les valeurs azotées moyennes du fourrage frais et du foin de 60 jours sont significativement plus élevées $(\mathrm{p}<0,05)$ que celles des foins de 75 et de 90 jours et au sein de chacun des deux (2) groupes de moyennes, aucune différence significative n'est observée. Quant aux valeurs énergétiques, celle du fourrage frais est significativement supérieure à celles des trois (3) foins et celle du foin de 75 jours est significativement supérieure à celle du foin de 60 jours. Le fourrage frais apparaît ainsi plus énergétique que le foin de 75 jours, le plus énergétique des foins. Il est suivi dans l'ordre par les foins de 90 et 60 jours.

\section{Rendements en valeurs azotée et énergétique}

Les rendements en valeur azotée (PBD) et en énergie (UF) sont consignés dans le Tableau 4. Les rendements moyens en valeur azotée ont baissé régulièrement, mais pas de manière significative, du fourrage frais aux foins et entre les foins, du foin de 60 jours et à celui de 90 jours. Entre les rendements moyens énergétiques des quatre (4) types de fourrages, aucune différence significative n'est notée. Le rendement en énergie du foin de 60 jours a été plus faible, suivi dans l'ordre par ceux du fourrage frais et des foins de 75 et 90 jours.

Tableau 1: Rendements moyens en fourrage.

\begin{tabular}{lc}
\hline \multicolumn{1}{c}{ Fourrages } & Rendements (t MS/ ha) \\
\hline Fourrage frais (79-84 jours) & $1,19 \pm 0,60$ \\
Fourrage sec (foin 60 jours) & $1,16 \pm 0,57$ \\
Fourrage sec (foin 75 jours) & $1,49 \pm 0,48$ \\
Fourrage sec (foin 90 jours) & $1,50 \pm 0,79$ \\
\hline
\end{tabular}

MS : matière sèche. 
Tableau 2: Teneurs moyennes en nutriments des fourrages.

\begin{tabular}{|c|c|c|c|c|}
\hline \multirow[b]{2}{*}{ Nutriments } & \multicolumn{4}{|c|}{ Fourrages } \\
\hline & FF79-84 & F60 & F75 & F90 \\
\hline MS (\%) & $93,54 \pm 0,70^{b}$ & $93,78 \pm 0,10^{a b}$ & $94,40 \pm 0,17^{\mathrm{a}}$ & $93,92 \pm 0,28^{a b}$ \\
\hline CT (\% MS) & $7,03 \pm 0,43 \quad \mathrm{c}$ & $9,23 \pm 1,05^{\mathrm{a}}$ & $7,36 \pm 0,30 \mathrm{bc}$ & $8,31 \pm 0,38^{a b}$ \\
\hline $\mathrm{MO}(\% \mathrm{MS})$ & $86,51 \pm 0,53^{a b}$ & $84,55 \pm 1,07 \quad c$ & $87,04 \pm 0,24^{a}$ & $85,60 \pm 0,17^{b}$ \\
\hline $\mathrm{PB}(\% \mathrm{MS})$ & $19,56 \pm 0,43^{\mathrm{a}}$ & $18,65 \pm 0,27^{\mathrm{b}}$ & $15,16 \pm 0,77 \mathrm{c}$ & $14,16 \pm 0,38$ \\
\hline $\mathrm{CB}(\% \mathrm{MS})$ & $27,26 \pm 1,93^{\mathrm{b}}$ & $34,93 \pm 1,52^{a}$ & $35,27 \pm 0,54^{\mathrm{a}}$ & $33,82 \pm 0,68^{a}$ \\
\hline NDF (\% MS) & $68,66 \pm 2,00^{\mathrm{a}}$ & $64,74 \pm 0,80^{b}$ & $64,99 \pm 0,54^{b}$ & $65,02 \pm 0,68^{b}$ \\
\hline $\mathrm{ADF}(\% \mathrm{MS})$ & $33,84 \pm 1,24^{b}$ & $40,10 \pm 0,73^{a}$ & $40,96 \pm 0,59^{a}$ & $40,32 \pm 0,74^{\mathrm{a}}$ \\
\hline $\mathrm{EE}(\% \mathrm{MS})$ & $1,67 \pm 0,06^{\mathrm{b}}$ & $1,81 \pm 0,06^{\mathrm{a}}$ & $1,49 \pm 0,06 \quad \mathrm{c}$ & $1,33 \pm 0,04 \quad d$ \\
\hline ENA (\% MS) & $38,02 \pm 1,69^{a}$ & $29,16 \pm 0,73 \mathrm{c}$ & $35,12 \pm 0,81^{b}$ & $36,75 \pm 0,59^{a b}$ \\
\hline
\end{tabular}

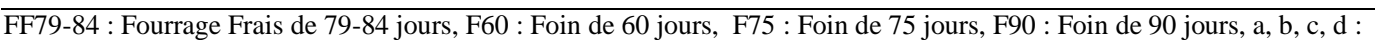
des lettres différentes affectées aux moyennes au sein d'une même ligne indiquent des différences significatives entre les groupes $(\mathrm{p}<0,05)$, MS : matière sèche, $\mathrm{CT}$ : centres totales, $\mathrm{MO}:$ matière organique, $\mathrm{PB}$ : protéine brute, $\mathrm{CB}:$ cellulose brute, NDF : neutral detergent fiber, ADF : acid detergent fiber, EE : extrait éthéré, ENA : extractif non azoté.

Tableau 3: Valeurs azotée et énergétique moyennes des fourrages.

\begin{tabular}{lcc}
\hline \multicolumn{1}{c}{ Fourrages } & PBD $(\mathbf{g} / \mathbf{~ k g ~ M S})$ & UF $(/ \mathbf{~ k g ~ M S})$ \\
\hline Fourrage frais (79-84 jours) & $150,65 \pm 0,43^{\mathrm{a}}$ & $0,70 \pm 0,00^{\mathrm{a}}$ \\
Fourrage sec (foin 60 jours) & $141,48 \pm 0,27^{\mathrm{a}}$ & $0,67 \pm 0,01^{\mathrm{c}}$ \\
Fourrage sec (foin 75 jours) & $106,60 \pm 0,77^{\mathrm{b}}$ & $0,69 \pm 0,00^{\mathrm{b}}$ \\
Fourrage sec (foin 90 jours) & $96,60 \pm 0,38^{\mathrm{b}}$ & $0,68 \pm 0,01^{\mathrm{bc}}$ \\
\hline
\end{tabular}

$\overline{\mathrm{PBD}}$ : protéine brute digestible, UF : unité fourragère, MS : matière sèche, $\mathrm{a}, \mathrm{b}, \mathrm{c}:$ des lettres différentes affectées aux moyennes au sein d'une même colonne indiquent des différences significatives entre les groupes $(\mathrm{p}<0,05)$.

Tableau 4: Rendements moyens en valeurs azotée et énergétique.

\begin{tabular}{lcc}
\hline Fourrages & PBD $(\mathbf{k g} / \mathbf{h a})$ & UF/ ha \\
\hline Fourrage frais (79-84 jours) & $180 \pm 90$ & $834 \pm 417$ \\
Fourrage sec (foin 60 jours) & $164 \pm 81$ & $787 \pm 391$ \\
Fourrage sec (foin 75 jours) & $158 \pm 51$ & $1025 \pm 331$ \\
Fourrage sec (foin 90 jours) & $145 \pm 77$ & $1023 \pm 540$ \\
\hline
\end{tabular}

PBD : protéine brute digestible, UF : unité fourragère. 


\section{DISCUSSION}

\section{Rendements moyens en fourrage}

Les rendements obtenus (Tableau 1) sont globalement faibles voire très faibles comparés à la plupart des données de la littérature. La physionomie de la saison des pluies 2018 dans le CNBAS expliquerait en partie ces résultats. En effet, elle se caractérise par une installation tardive ( 1 ère pluie utile le 23 août) et un cumul annuel de $503 \mathrm{~mm}$. Ce dernier apparaît relativement faible comparé à la pluviométrie annuelle (650 à $2500 \mathrm{~mm}$ ) dont a besoin le Mucuna pour bien se développer. Pour cette zone à pluviométrie relativement faible, l'étude a été menée dans des conditions pluviométriques difficiles pour le Mucuna. Néanmoins, nos rendements sont conformes à certains résultats rapportés dans la littérature par Adjolohoun et al. (2008), SidibéAnago et al. (2009) et Kanwe et al. (2015), qui varient entre 0,90 et 1,71 t MS/ha. Nos résultats sont aussi légèrement en dessous de celui obtenu par Nyambati et Sollenberger (2003), qui est de 2,06 t MS/ha et de celui rapporté par Houndjo et al. (2018), qui est de 2,00 t MS/ha. Nos rendements sont par contre très faibles comparés à la plupart des résultats rapportés dans la littérature par Diaz et al. (2000), Asongwed-Awa et Onana (2003), Eilittä et al. (2003), Mbuthia et Gachuiri (2003), SidibéAnogo et al. (2009), Ecocrop (2011), Klein et al. (2014), Heuzé et al. (2015) et Houndjo et al. (2018), lesquels varient entre 3,07 et $16,40 \mathrm{t}$ MS/ha.

\section{Compositions chimiques moyennes des fourrages}

Les teneurs en nutriments des fourrages (Tableau 2) ont varié entre les types de fourrages et suivant les temps de croissance. Les teneurs en protéines brutes (PB) sont plus élevées et plus basses respectivement pour le fourrage frais de 79 à 84 jours et le foin de 90 jours. Les processus de fenaison au champ, de ramassage, de transport au moyen d'une charrette à l'âne et de stockage en vrac des foins dans le magasin de fourrage expliqueraient les différences observées entre le fourrage frais de 79 à 84 jours et les trois (3) types de foins de 60, 75 et 90 jours. Par contre, entre les foins, les temps de croissance semisfauche expliqueraient les différences notées. Les teneurs obtenues confirment aussi les teneurs élevées en $\mathrm{PB}$ des légumineuses fourragères comparées aux graminées et leur relative stabilité durant leur cycle de développement (César et al., 2004 cité par Dimon et al., 2018). Jusqu'à trois (3) mois d'âge, le fourrage frais et les foins ont eu des teneurs relativement élevées en PB. Nos résultats sont légèrement supérieurs à ceux obtenus par Nantoumé et al. (2000), Kiema et al. (2012), Bahini et al. (2016) et Houndjo et al. (2018), qui sont compris entre 8,47 et $12,21 \%$ MS. Ils sont pour la plupart proches de ceux obtenus par Adjorlolo et al. (2001 et 2004), Muinga et al. (2003), Nyambati et Sollenberger (2003), Babatounde et al. (2010) et Mahaman et al. (2017), qui se situent entre 14,02 et 20,00\% MS. Nos teneurs en PB sont par contre relativement faibles comparées à celles indiquées dans la littérature, 23,16 à 26,00\% MS par Aboh et al. (2002), Houndjo et al. (2018) et Nkana et al. (2020).

Les teneurs en $\mathrm{CB}$ et en ADF du fourrage frais sont moins élevées que celles des foins. Et c'est l'inverse pour les teneurs en NDF. Entre les foins, les différences entre les teneurs en $\mathrm{CB}$, ADF et NDF sont moindres. Les différences nettes observées entre le fourrage frais et les foins seraient dues aux processus de constitution des foins qui ont conduit à des accroissements des constituants pariétaux (CB et ADF). Par contre, les temps de croissance n'ont pas entrainé des hausses sensibles des teneurs en $\mathrm{CB}$ et ADF ni des baisses importantes de celles en NDF des foins. Pour la cellulose brute $(\mathrm{CB})$, nos résultats sont légèrement plus élevés que ceux indiqués par Kiema et al. (2012) et Mahaman et al. (2017), qui sont compris entre 23,12 et $24,49 \%$ MS. Nos résultats corroborent globalement ceux de la littérature, 26,30 à 37,50\% MS et rapportés par Nantoumé et al. (2000), Adjorlolo et al. (2001 et 2004), Aboh et al. (2002), Kiema et al. (2012), Bahini et al. (2016), Mahaman et al. (2017) et Nkana et al. (2020). Concernant NDF, nos résultats relativement élevés, ne corroborent pas certains de la littérature, 37,40$54,40 \%$ MS, obtenus par Adjorlolo et al. (2001 
et 2004), Mbuthia et Gachuiri (2003), Muinga et al. (2003) et Nyambati et Sollenberger (2003). Ils ne sont cependant pas exceptionnellement élevés comparés à celui de 71,56\% MS obtenu par Bahini et al. (2016). Pour ADF, nos résultats sont globalement conformes à ceux rapportés dans la littérature, 34,00 à 41,60\% MS, par Adjorlolo et al. (2001 et 2004), Mbuthia et Gachuiri (2003) et Babatounde et al. (2010). Ils sont inférieurs au résultat obtenu par Bahini et al. (2016), qui est de $58,41 \%$ MS.

\section{Valeur nutritive des fourrages}

Les valeurs azotées (PBD) des fourrages frais (79 à 84 jours) et du foin de 60 jours sont nettement plus élevées que celles des foins de 75 et 90 jours (Tableau 3). Entre les foins, les valeurs azotées ont baissé sensiblement du foin de 60 jours à ceux de 75 et 90 jours et légèrement entre les foins de 75 et 90 jours. Entre le fourrage frais et les foins de 75 et 90 jours, les processus de constitution des foins expliqueraient les différences élevées observées. Ils seraient également à l'origine de la différence légère notée entre le fourrage frais et le foin de 60 jours. Entre les foins, les temps de croissance semis-fauche expliqueraient les différences élevées et légères notées respectivement entre le foin de 60 jours et les foins de 75 et 90 jours et entre les foins de 75 et 90 jours. Les valeurs azotées des fourrages sont nettement supérieures à celles obtenues par Kiema et al. (2012), qui sont 43,44 et 62,90 $\mathrm{g} / \mathrm{kg}$ MS. Elles sont comprises dans celles rapportées pour les légumineuses fourragères par César et al. (2004) cité par Dimon et al. (2018), qui se situent entre 78 et $199 \mathrm{~g} / \mathrm{kg}$ MS. Concernant les valeurs énergétiques (UF), le fourrage frais est apparu nettement plus énergétique que les foins. Les processus de fenaison expliqueraient les différences notées. Entre les foins, celui de 60 jours a été sensiblement moins énergétique que celui de 75 jours et légèrement moins énergétique que celui de 90 jours. Entre les foins de 75 et de 90 jours, une légère supériorité a été notée en faveur du foin de 75 jours. Les temps de croissance de ces foins ne permettent pas d'expliquer les différences observées. Pour les valeurs énergétiques, nos résultats sont légèrement en dessous de ceux obtenus par Kiema et al. (2012), qui sont 0,83 et $0,93 \mathrm{UF} / \mathrm{kg}$ MS. Ils traduisent ainsi des fourrages moins énergétiques.

\section{Conclusion}

Notre étude avait pour objectif d'évaluer la production et la valeur nutritive des fourrages frais et secs de M. pruriens cv. Ghana dans le Centre et Nord bassin arachidier sénégalais. Les résultats obtenus (rendements en fourrage, PBD et UF, compositions chimiques et valeurs azotée et énergétique) démontrent que la culture fourragère du $M$. puriens $\mathrm{cv}$. Ghana est possible dans le Centre et Nord bassin arachidier sénégalais. Elle peut ainsi contribuer à atténuer le déficit fourrager et à la sécurité alimentaire des ruminants domestiques. En effet, les productions obtenues s'inscrivent dans les limites de celles rapportées par d'autres auteurs. Par ailleurs, le fourrage frais, même âgé de trois (3) mois est plus riche en énergie et en protéines que le foin de 60 jours. Dès lors, à défaut d'un pâturage direct, la fauche et la distribution à l'auge du fourrage frais constituent la meilleure forme d'exploitation. S'agissant de l'exploitation et de la conservation en foin, la récolte du fourrage âgé de 75 jours est la mieux indiquée. Ces résultats mettent en évidence une certaine flexibilité pour la récolte du fourrage qui peut se faire jusqu'à 90 jours, soit en fin de saison des pluies, moment où la gestion de la main d'œuvre familiale et du calendrier des récoltes se pose avec acuité.

\section{CONFLIT D'INTERETS}

Les auteurs déclarent qu'ils n'ont aucun conflit d'intérêts.

\section{CONTRIBURIONS DES AUTEURS}

$\mathrm{AD}$ a supervisé les travaux de terrain, les analyses chimiques, la rédaction, a lu et commenté, suggéré, corrigé par écrit. Après l'intégration des contributions des autres auteurs, il a encore relu et corrigé en vue de la finalisation du manuscrit. SN a contribué par la lecture et des commentaires, suggestions, corrections écrits avec un accent particulier sur 
tout ce qui a trait aux statistiques. MTD et TDTN ont contribué à travers la lecture et des commentaires, suggestions, corrections écrit.

\section{REFERENCES}

Aboh AB, Olaafa M, Dossou-Gbété GSO, Dossa AD, Djagoun N. 2002. Ingestion volontaire et digestibilité apparente d'une ration à base de la farine de graines de Mucuna pruriens var. utilis complété de fourrages chez les lapins. Tropicultura, 20(4): 165-169.

Adjolohoun S, Buldgen A, Adandedjan C, Decruyenaere V, Dardenne P. 2008. Yield and nutritive value of herbaceous and browse forage legumes in the Borgou region of Benin. Tropical Grasslands, 42: 104-111.

Adjorlolo LK, Amaning-Kwarteng K, Fianu FK. 2004. Preference of sheep for three forms of Mucuna forage and the effect of supplementation with Mucuna forage in the performance of sheep. Trop. Anim. Health Prod., 36(2): 145-156. DOI: 10.1023/b:trop.0000012102.42938.90

Adjorlolo LK, Amaning-Kwarteng K, Fianu FK. 2001. In vivo digestibility and effect of supplemental Mucuna forage on treated rice straw degradation. Small Rum. Res., 41(3): 239-245. DOI: 10.1016/S0921-4488(01)00221-8

AOAC (Association of Official Analytical Chemists). 1995. Official Methods of Analysis $\left(16^{\text {th }}\right.$ edn). AOAC: Arlington, Va, USA.

Asongwed-Awa A, Onana J. 2003. Variability in productivity of Mucuna pruriens varieties in semi-arid environment, p. 6. https://hal.archives-ouvertes.fr/hal00137962

Babatounde S, Oumourou M, Tchabi VI, Lecomte T, Houinato M, Adandedjan C. 2010. Ingestion volontaire et préférences alimentaires chez des moutons Djallonké nourris avec des graminées et des légumineuses fourragères tropicales cultivées au Bénin. Int. J. Biol. Chem. Sci., 4(4): 1030-1043. DOI: 10.4314/ijbcs.v4i4.63041
Bahini MJD, Babatounde S, Aboh A, Saïdou A, Kindomihou V, Mensah GA. 2016. Production de gaz in vitro et valeur nutritive des fanes de seize variétés à usage multiple de niébé (Vigna unguiculata $\mathrm{L}$, Walp) cultivées au Bénin. J. Appl. Biosc., 105 : 10134-10151. DOI: http://dx.doi.org/10.4314/jab.v105i1.10

César J, Ehouinsou M, Gouro A. 2004. Production fourragère en zone tropicale et conseils aux éleveurs : conseils et formation en appui à la production laitière. S.l.: s.n., $\quad$ p. 48. https://agritrop.cirad.fr/556496

Coulibaly D, Ba A, Koné AK. 2018. Culture du mucuna (Mucuna pruriens) pour la production de fourrage en zone sud du Mali. In Guides Méthodologiques Fiches Techniques: Innovations pour l'Amélioration de la Productivité et de la Durabilité des Systèmes d'Exploitation à base de Coton au Mali, Soumaré M, Havard M. (eds). IER, CIRAD, AFD, CNRA: Bamako, Mali; 19-22.

Coulibaly K, Vall E, Autfray P, Sedogo PM. 2012. Performance technico-économique des associations maïs/niébé et maïs/mucuna en situation réelle de culture au Burkina Faso: potentiels et contraintes. Tropicultura, 30(3): 147154.

Dagnelie P. 1998. Statistique Théorique et Appliquée. Tome 1. Statistique Descriptive et Bases de l'Inférence Statistique. De Boeck Université: Paris, Bruxelles.

Dahouda M. 2009. Contribution à l'étude de l'alimentation de la pintade locale au Bénin et perspectives d'amélioration à l'aide de ressources non conventionnelles. Thèse de doctorat. Université de Liège (ULg), Liège, Belgique, p. 174.

Diaz MF, Gonzales A, Mora C, Curbelo F. 2000. Leaf protein concentration from Styzolobium aterimum. Cuba. J. Agric. Sci., 34(2): 171-177.

Dijkstra ND. 1957. Research into the digestibility and feeding value of some 
grass species and grass leys. Verls Landb. Onderz., 1: 63.

Dimon E, Idrissou Y, Soule AH, Assani SA, Assogba BCG, Toukourou Y, Attakpa EY, Alkoiret IT, Mensah GA. 2018. Synthèse des connaissances sur la valorisation des légumineuses fourragères dans l'alimentation des ruminants élevés au Bénin. Bulletin de la Recherche Agronomique du Bénin, 84: 43-51.

Dione M, Diop O, Dieye PN, Ba DN, Ndao B. 2008. Caractérisation et typologie des exploitations agricoles familiales $\mathrm{du}$ Sénégal: Bassin arachidier. Etudes et documents, Vol. $8 \quad \mathrm{~N}^{\circ} 3$. Institut Sénégalais de Recherches Agricoles (ISRA): Dakar, Sénégal.

ECHO (Educational Concerns for Hunger Organization). 2020. Pois Mascate volubile: pois du bengale, Мисиna pruriens. ECHO Fiche d'informations de la plante: Floride, USA.

Ecocrop. 2011. Ecocrop database. FAO. https://www.feedipedia.org/node/5368

Eilittä M, Sollenberger I E, Littell RC, Harrington LW. 2003. On-farm experiments with maize-Mucuna systems in the Los Tuxtlas Region of Veracruz, Mexico. I. Mucuna biomass and maize grain yield. Exp. Agric., 39(1): 5-17.

DOI: https://doi.org/10.1017/S0014479702001 126.

Heuzé V, Tran G, Hassoun P, Renaudeau D, Bastianelli D. 2015. Velvet bean (Mucuna pruriens). Feedipedia, a programme by INRA, CIRAD, AFZ and FAO.

http://www.feedipedia.org/node/270.

Houndjo DBM, Adjolohoun S, Gbenou B, Saidou A, Ahoton L, Houinato M, Seibou Toleba S, Sinsi BA. 2018. Sociodemographic and economic characteristics, crop-livestock production systems and issues for rearing improvement: A review. Int. J. Biol. Chem. Sci., 12(1): 519-541. DOI: https://dx.doi.org/10.4314/ijbcs.v12i1.41
Kanwe A, Dabire D, Toe P, Ouédraogo S, Havard M. 2015. Rapport de synthèse des résultats au Burkina Faso. Projet: Conception de systèmes de production innovants à base de mucuna et autres cultures fourragères (CIMF). ASAP, CIRDES, INERA, IDR/UPB, CIRAD : Ouagadougou, Burkina Faso.

Kiema A, Sawadogo I, Ouédraogo T, Nianogo AJ. 2012. Stratégies d'exploitation du fourrage par les éleveurs de la zone sahélienne du Burkina Faso. Int. J. Biol. Chem. Sci., 6(4): 1492-1505. DOI : http://dx.doi.org/10.4314/ijbcs.v6i4.8

Klein HD, Rippstein G, Hugueni J, Toutain B, Guerin H, Louppe D. 2014. Les Cultures Fourragères. Collection Agricultures Tropicales en poche. Editions Quæ, CTA. Presses Agronomiques de Gembloux: Gembloux, Belgique.

Mahaman MMO, Chaibou M, Abdou D, Abdou Ide B, Issoumane Sitou M, Mani M. 2017. Evaluation de l'ingestibilité des bouchons alimentaires à base des sousproduits de niébé utilisés pour l'engraissement des ovins. Revue des BioRessources, 7(2): 1-11.

Mbuthia EW, Gachuiri CK. 2003. Effect of inclusion of Mucuna pruriens and Dolichos lablab forage in Napier grass silage on silage quality and voluntary intake and digestibility in sheep. Trop. Subtrop. Agroecosyst., 1(2-3): 123-128.

Muinga RW, Sala HM, Mureithi JG. 2003. The effect of Mucuna (Mucuna pruriens) forage on the performance of lacting cows. Trop. Ssubtrop. Agroecosyst., 1(23): 87-91.

Nantoumé H, Kouriba A, Togola D, Ouologuem B. 2000. Mesure de la valeur alimentaire de fourrage et de sousproduits utilizes dans l'alimentation des petits ruminants. Revue Elev. Méd. Vét. Pays Trop., 53(3): 279-284. DOI: https://doi.org/10.19182/remvt.9725

Nkana JGK, Mweugang NN, Germanus SB, Semi YA, Ntsafack P, Awantu C, Wangba CT, Noumbissi MNB, Djoumessi FGT, Miégoué E. 2020. Effet de quelques légumineuses sur la 
reproduction, la croissance et la carcasse chez les cochons d'inde (Cavia porcellus L.). Int. J. Biol. Chem. Sci., 14(2): 600612.

DOI:

https://dx.doi.org/10.4314/ijbcs.v14i2.23

Nyambati EM, Sollenbeger LE. 2003.

Nutritive value of top-canopy herbage of Mucuna and lablab relay cropped in maize in sub-highlands of northwestern Kenya. Trop. Subtrop. Agroecosyst., 1(23): 81-86.

Padmesh P, Reji JV, Jinish Dhar M, Seeni S. 2006. Estimation of genetic diversity in varieties of Mucuna pruriens using RAPD. Biologia Plantarum, 50(3): 367372. DOI : $10.1007 / \mathrm{s} 10535-006-0051-\mathrm{z}$

Rivière R. 1978. Manuel d'alimentation des ruminants domestiques en milieu tropical. Manuels et Précis d'Elevage $\mathrm{N}^{\circ} 9\left(2^{2 \mathrm{ème}}\right.$ ed). IEMVT: Paris, France.

Sidibé-Anago AG, Ouedraogo GA, Kanwé AB, Ledin I. 2009. Foliage yield, chemical composition and intake charactistics of three Mucuna varieties. Trop. Subtrop. Agroecosyt., 1(10): 7584.
St-Laurent L, Livsey J, Arnason JT, Bruneau A. 2002. Variation in L-Dopa concentration in accessions of Mucuna pruriens (L.) DC. and Mucuna brachycarpa Rech. In Food and Feed from Mucuna: Current Uses and the Way Forward: Proceedings of an International Workshop. Tegucigalpa, Hondras, April, 26-29, 2000. Milton Flores, Milton Flores B., International Cover Crop Clearinghouse, Center for Cover crops Information and Seed Exchange in Africa, World Hunger Research Center (ed). International Cover Crops Clearinghouse: Tegucigalpa; 325-373.

Van Soest PJ. 1965. Symposium of factors influencing the volontary intake of herbage by ruminants: volontary intake in relation to chemical composition and digestibility. J. Anim. Sci., 24(3) : 834843.

DOI : https://doi.org/10.2527/jas1965.243834x. 